\title{
A modified Poisson-Boltzmann analysis of the capacitance behavior of the electric double layer at low temperatures
}

\author{
Douglas Henderson
}

L. B. Bhuiyan

C. W. Outhwaite

Follow this and additional works at: https://scholarsarchive.byu.edu/facpub

Part of the Biochemistry Commons, and the Chemistry Commons

\section{Original Publication Citation}

Bhuiyan, L. B., C. W. Outhwaite, and D. Henderson."A modified Poisson-Boltzmann analysis of the capacitance behavior of the electric double layer at low temperatures." The Journal of Chemical Physics 123 (25).

\section{BYU ScholarsArchive Citation}

Henderson, Douglas; Bhuiyan, L. B.; and Outhwaite, C. W., "A modified Poisson-Boltzmann analysis of the capacitance behavior of the electric double layer at low temperatures" (2005). Faculty Publications. 359. https://scholarsarchive.byu.edu/facpub/359 accepted for inclusion in Faculty Publications by an authorized administrator of BYU ScholarsArchive. For more information, please contact ellen_amatangelo@byu.edu. 


\title{
A modified Poisson-Boltzmann analysis of the capacitance behavior of the electric double layer at low temperatures
}

\author{
L. B. Bhuiyan ${ }^{\text {a) }}$ \\ Laboratory of Theoretical Physics, Department of Physics, University of Puerto Rico, \\ San Juan 00931-3343, Puerto Rico \\ C. W. Outhwaite \\ Department of Applied Mathematics, University of Sheffield, Sheffield S3 7RH, United Kingdom \\ D. Henderson \\ Department of Chemistry and Biochemistry, Brigham Young University, Utah 84602-5700
}

(Received 25 April 2005; accepted 9 June 2005; published online 26 July 2005)

\begin{abstract}
The modified Poisson-Boltzmann theory is used to analyze the anomalous behavior of the electric double layer capacitance for small surface charge at low temperatures and densities. Good agreement is found with simulation and recent density-functional theory results. Negative adsorption is also found in line with theory and simulation. An unsatisfactory feature is the relatively poor structure in this region due to the inherent approximations in the theory. This feature is unimportant in relation to the capacitance results but has implications when calculating adsorption properties. (C) 2005 American Institute of Physics. [DOI: 10.1063/1.1992427]
\end{abstract}

\section{INTRODUCTION}

Until recently there appeared to be a contradiction between the molten salt/electrode and electrolyte/electrode double layer capacitance results for small surface charge. The capacitance for molten salts increased with temperature while that of the electrolyte always apparently decreased, which suggested that the different behavior arose from the higher ionic density of the molten salt. ${ }^{1-3}$ However the simulation results of Boda et al. ${ }^{4,5}$ and Henderson ${ }^{6}$ using the restricted primitive model (RPM) electrolyte next to a hard wall at low surface charge have reconciled the two capacitance results. At low temperatures and densities the electrolyte capacitance increases with temperature, in an analogous fashion to that for the molten salt, while having a negative slope at higher temperatures. Because the relative permittivity or dielectric constant of a molten salt is small (near unity), the "effective" or reduced temperature of a molten salt is low even though the ambient temperature is high. Thus the difference in the molten salt and electrolyte capacitances is a temperature rather than a density effect.

Ionic association occurs at low reduced temperatures. Using the concept of ionic association in conjunction with the mean spherical approximation (MSA), Holovko et al. ${ }^{7}$ were able to give an explanation of the low-temperature electrolyte capacitance behavior, where earlier the GouyChapman (GC), MSA, generalized MSA, and various density-functional theories had failed. ${ }^{4-6,8-10}$ Recently a new version of the density-functional theory (DFT) has been investigated, which successfully reproduces the simulation capacitance results besides predicting satisfactory structural properties. ${ }^{11}$ We show here that the modified PoissonBoltzmann (MPB) theory ${ }^{12-14}$ can also predict the correct

a)Electronic mail: beena@beena.cnnet.clu.edu qualitative capacitance behavior at low temperatures and densities. The MPB theory uses an electrostatic potential approach based on the Born-Green-Kirkwood-Yvon hierarchy to correct the mean-field GC theory, and is known to successfully reproduce the structural and thermodynamic electric double layer properties of a RPM electrolyte. ${ }^{12-14}$

\section{THEORY}

The electric double layer is modeled by a RPM electrolyte next to a plane hard wall with uniform surface charge density $\sigma$, the electrolyte being characterized by ions $i$ of charge $e_{i}$ and diameter $d$ moving in a dielectric medium of permittivity $\varepsilon$. The electrode is taken to have the same permittivity $\varepsilon$ so that there is no imaging. In the diffuse layer the mean electrostatic potential $\psi(x)$ satisfies Poisson's equation

$$
\frac{d^{2} \psi}{d x^{2}}=-\frac{4 \pi}{\varepsilon} \sum_{i} \lambda_{i}|e| \rho_{i} g_{i}(x)
$$

where $x$ is the perpendicular distance into the solution from the electrode, $\rho_{i}$ is the mean number density of ions of type $i$, $e_{i}=\lambda_{i}|e|$ with $\lambda_{i}$ the ion valency and $|e|$ the magnitude of the electron charge, and $g_{i}(x)$ is the wall-ion distribution function. As ions are excluded from the region $0<x \leqslant d / 2$, Eq. (1) is then Laplace's equation in this region with solution

$$
\psi(x)=\psi(0)-4 \pi \sigma x / \varepsilon .
$$

To formulate an equation for $\psi(x)$, where $x>d / 2$, we use the MPB5 expression for $g_{i}(x)$ in Poisson's equation, namely, 


$$
g_{i}(x)=\xi_{i}(x) \exp \left[-\frac{\beta e_{i}^{2}}{2 \varepsilon d}\left(F-F_{0}\right)-\beta e_{i} L(\psi)\right] .
$$

Here $\xi_{i}(x)=g_{i}\left(x \mid e_{i}=0\right)$ is the exclusion volume term, $\beta$ $=1 / k T$ with $k$ being Boltzmann's constant and $T$ the absolute temperature, and

$$
\begin{aligned}
& F=\frac{4}{[4+\kappa(d+2 x)]}, \quad d / 2 \leqslant x \leqslant 3 d / 2, \\
& F=\frac{1}{(1+\kappa d)}, \quad x \geqslant 3 d / 2, \\
& \kappa^{2}=(4 \pi \beta / \varepsilon) \sum_{i} e_{i}^{2} \rho_{i} g_{i}(x), \\
& \kappa_{0}=\lim _{x \rightarrow \infty} \kappa, \quad F_{0}=\lim _{x \rightarrow \infty} F=\left(1+\kappa_{0} d\right)^{-1},
\end{aligned}
$$$$
L(\psi)=\frac{F}{2}[\psi(x+d)+\psi(x-d)]-\frac{(F-1)}{2 d} \int_{x-d}^{x+d} \psi(X) d X,
$$

with

$$
\begin{aligned}
\xi_{i}(x)= & H(x-d / 2) \exp \left\{-2 \pi \int_{\infty}^{x} \sum_{j} \rho_{j} \int_{\max (d / 2, y-d)}^{y+d}\right. \\
& \left.\times(X-y) g_{j}(X) \exp \left[-\beta e_{j} \Phi(y)\right] d X d y\right\} .
\end{aligned}
$$

In Eq. (7), $H(x)$ is the Heaviside unit step function and $\Phi(y)$ is the fluctuation potential for the discharged ion $i$ evaluated over the surface $S$ of the exclusion volume $V$ of $i$ at $y$, and is given by

$$
\Phi(y)=\frac{F}{4 \pi d} \int_{V} \frac{d^{2} \psi}{d x^{2}} d V, \quad y \geqslant d / 2 .
$$

The MPB5 equation is based on Loeb's closure in the Kirkwood hierarchy of equations with Eq. (7) being derived from the Born-Green-Yvon hierarchy. ${ }^{15}$ In the rest of the paper, the above MPB5 system of equations will be referred to as the MPB equation. An alternative exclusion volume term of comparable accuracy is ${ }^{15,16}$

$$
\xi_{i}(x)=\xi_{i}(\sigma=0) \exp \left\{\int_{V} \sum_{j} \rho_{j} c_{i j}^{0}\left[g_{j}(x)-g_{j}(x \mid \sigma=0)\right] d V\right\},
$$

where $c_{i j}^{0}$ is the inhomogeneous neutral sphere direct correlation function, which can be approximated by the direct correlation function for bulk hard spheres. For the parameters treated here the two exclusion volume terms are close to unity and lead to similar predictions for both structural and thermodynamic properties. Earlier MPB equations ${ }^{15,17}$ are less accurate as more approximate expressions were used for $F$ or $\xi_{i}(x)$. If required, the MPB equation can be adapted to treat imaging. ${ }^{12}$ The classical GC theory is derived by putting $\xi_{i}(x)=H(x)$ and $F=F_{0}$ and letting $d \rightarrow 0$.
The MPB equation for $\psi(x)$ was solved numerically by using finite differences for the derivatives of $\psi(x)$ combined with a quasilinearization iteration procedure (see Refs. 12 and 17). This numerical technique is very efficient and robust and has been used successfully to solve the bulk MPB system of equations ${ }^{18}$ in the critical region. ${ }^{19,20}$ The associated boundary conditions for the potential problem are $\psi(x)$ and $d \psi / d x$ continuous in $x>0$, with $\psi(x)$ and $d \psi / d x \rightarrow 0$ as $x$ $\rightarrow \infty$. At the electrode surface we have $d \psi / d x=-4 \pi \sigma / \varepsilon$ which is implicit in the linear solution Eq. (2).

\section{RESULTS AND DISCUSSION}

Numerical solutions were found at small surface charge for the range of reduced parameters $T^{*}=\varepsilon d / \beta e^{2}, \rho^{*}=\left(\rho_{+}\right.$ $\left.+\rho_{-}\right) d^{3}=\rho d^{3}$, and $\sigma^{*}=\sigma d^{2} /|e|$ corresponding to those of the DFT calculations and the majority of the simulations of Refs. 4-6 and 11. The presented results are restricted to $T^{*}$ $\geqslant 0.07$ even though convergence can be achieved at the lowest simulation value of $T^{*}=0.06$. The restriction arises because MPB investigations of the RPM bulk electrolyte liquid-gas coexistence region gives, via the virial route, critical parameter values of $T^{*}=0.0635$ and $\rho^{*}=0.037 .{ }^{19,20}$ Consequently with $T^{*} \geqslant 0.07$ we expect to stay outside the MPB two-phase region. Similarly the DFT is restricted to $T^{*}$ $\geqslant 0.08$ because of the bulk MSA critical $T^{*}=0.0786$ calculated via the energy route. The best critical value simulation estimates are $T^{*}=0.0496$ and $\rho^{*}=0.079 .^{21}$
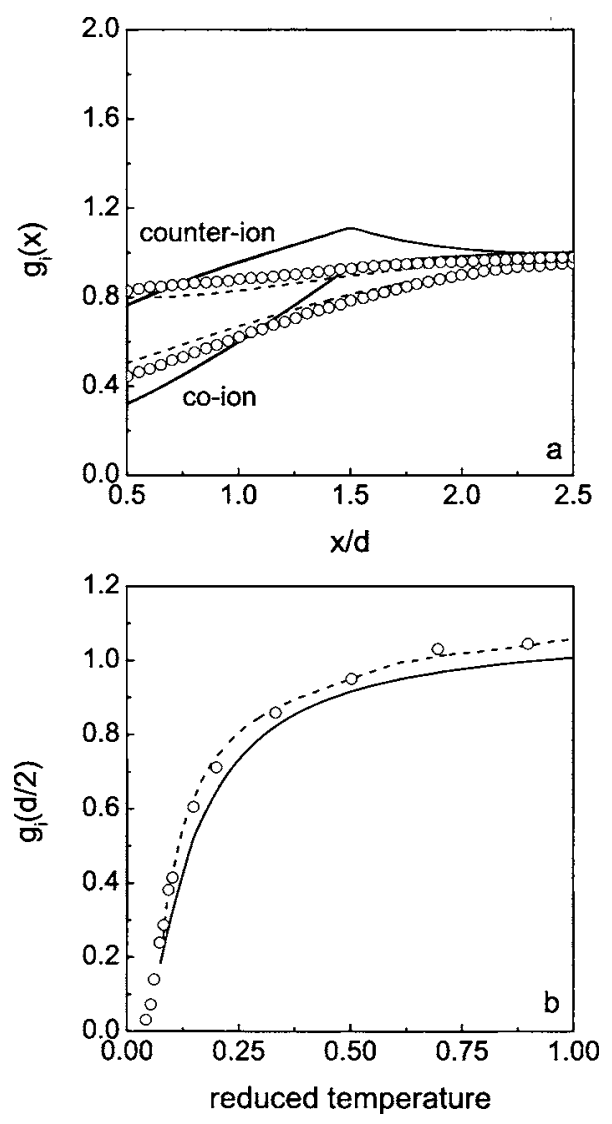

FIG. 1. (a) Coion and counterion $g_{i}(x)$ at $T^{*}=0.15, \rho^{*}=0.04$, and $\sigma^{*}$ $=0.00765$ (solid line, MPB; dashed line, DFT; open circles, simulation) (Ref. 5). (b) Variation of the contact value $g_{i}(d / 2)$ of the ion distribution functions with $T^{*}$ at $\sigma^{*}=0$ for $\rho^{*}=0.04$. The notation is the same as in (a). 


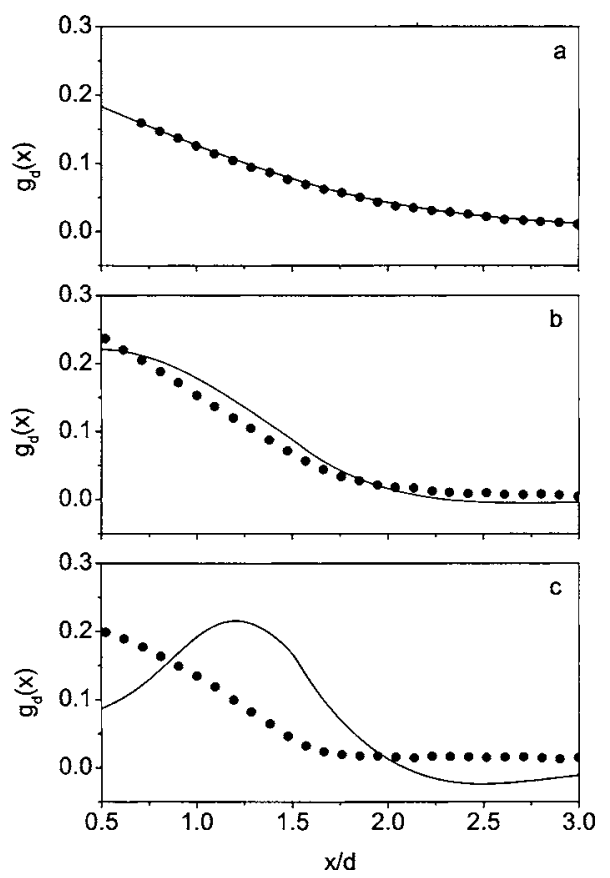

FIG. 2. The difference in the ion distribution functions $g_{d}=\left(g_{-}-g_{+}\right) / 2$ for $\rho^{*}=0.04$ and $\sigma^{*}=0.00765$ at (a) $T^{*}=0.5$, (b) $T^{*}=0.15$, and (c) $T^{*}=0.08$ (solid line, MPB; solid circles, simulation (Ref. 22)).

In Fig. 1(a) we compare the coion and counterion singlet distribution functions for the MPB, Monte Carlo (MC), and DFT at $\sigma^{*}=0.00765, \rho^{*}=0.04$, and $T^{*}=0.15$. All three approaches predict that both ion distributions are depleted near the wall with the coion reduction being the larger. However the MPB distributions have the unsatisfactory feature of a small maximum at $x=3 d / 2$ which arises from the approximate solution of the fluctuation problem in the neighborhood of the electrode. This unsatisfactory feature has been noted before at $\sigma^{*}=0$ for a metallic electrode. ${ }^{12}$ Comparison of $g_{d}\left(=\left(g_{-}-g_{+}\right) / 2\right)$ with simulation results ${ }^{22}$ in Fig. 2 indicates how the MPB structure begins to deviate at the lower $T^{*}$. To analyze the MPB predictions in Fig. 1, consider the distribution function given by Eq. (3). For symmetrical electrolytes at zero surface charge the mean potential $\psi(x)$ is zero so

$$
g_{i}(x)=\xi_{i}(x) \exp \left[-\frac{1}{2 T^{*}}\left(F-F_{0}\right)\right] .
$$

Thus for very small surface charge the two distribution functions are nearly identical and each split into essentially a product of a neutral term and an electrical term, the electrical term coming from ion-ion interactions. At the parameters of Fig. 1(a), the exclusion volume term $\xi_{i}(x) \sim 1$ for $x>d / 2$, so that $g_{i}$ is basically controlled by the positive sign of $F-F_{0}$. The condition $F>F_{0}$ arises from the wall distorting the atmosphere of ion $i$ and hence encouraging ionic association. As either of $\rho^{*}$ or $\sigma^{*}$ increases, $L(\psi)$ becomes dominant giving a positive counterion adsorption. The depletion of the singlet distribution functions is not restricted to low $T$. It has been seen in MPB and simulation work for 1:1 electrolytes at $\sigma=0$ with $d=4.25 \times 10^{-10} \mathrm{~m}, \varepsilon=78.5, T=298 \mathrm{~K}$, and electrolyte concentration $=0.1 \mathrm{~mol} \mathrm{dm}^{3}$ (corresponding to $\rho^{*}$ $=0.03$ and $\left.T^{*}=0.595\right){ }^{12,23}$ When $\sigma^{*}=0$ the ion distributions

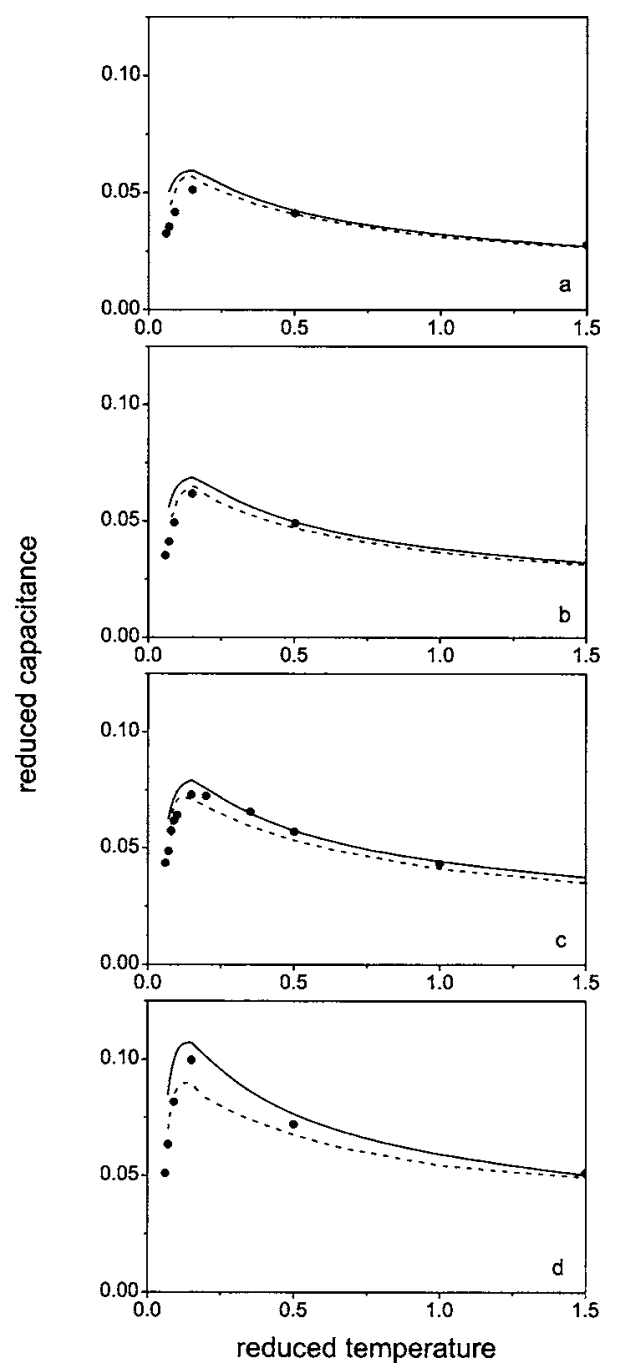

FIG. 3. Variation of the reduced integral capacitance $C_{i}^{*}$ with $T^{*}$ for (a) $\rho^{*}$ $=0.019$, (b) $\rho^{*}=0.028$, (c) $\rho^{*}=0.04$, and (d) $\rho^{*}=0.079$ (solid line, MPB; dashed line, DFT; solid circles, simulation (Ref. 5)).

are equal and the discontinuity in the slope is reduced. The change of the MPB contact value with temperature is then in good agreement with the simulation results [Fig. 1(b)].

The variation of the reduced integral capacitance $C_{i}^{*}$ $=C_{i} d / \varepsilon=\sigma / \psi(0)(d / \varepsilon)$ with temperature, for four values of $\rho^{*}$, is given in Figs. 3(a)-3(d). The reduced integral capacitance is used for simplicity, as for small $\sigma^{*}$ it is similar to the reduced differential capacitance $C_{d}^{*}=C_{d} d / \varepsilon=\partial \sigma / \partial \psi(0)(d / \varepsilon)$. An illustration of the difference between the MPB integral and differential capacitances is given in Fig. 4(b) at $T^{*}=0.1$ and $\rho^{*}=0.04$, corresponding to roughly the maximum $C_{i}^{*}$ in Fig. 3(c). There is little difference between $C_{i}^{*}$ and $C_{d}^{*}$ near zero surface charge so both capacitances are expected to show the same qualitative behavior in this region. For interest the integral and differential capacitances are shown in Fig. 4(a) at $T^{*}=0.595$ with $\rho^{*}=0.04$, which corresponds to an aqueous electrolyte at room temperature. We see that as $T^{*}$ is decreased to low values for fixed $\rho^{*}$, the variation between $C_{i}^{*}$ and $C_{d}^{*}$ increases for nonzero surface charge. In each of the Figs. 3(a)-3(d) the MPB and DFT capacitances have the same qualitative behavior as the simulation results. For small $T^{*}$ the capacitance slope is positive which changes to a nega- 


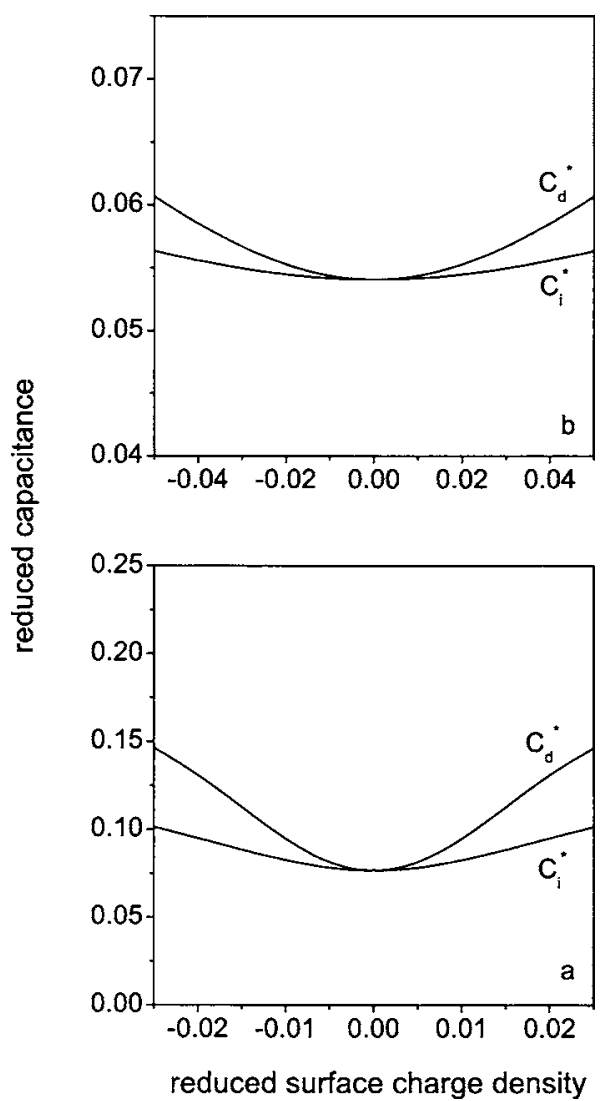

FIG. 4. Comparison of the MPB-reduced integral capacitance $C_{i}^{*}$ with the MPB-reduced differential capacitance $C_{d}^{*}$. (a) $T^{*}=0.595$ and $\rho^{*}=0.04$ and (b) $T^{*}=0.1$ and $\rho^{*}=0.04$.

tive slope as $T^{*}$ increases. The MPB theory predicts the positive slope for small $T^{*}$, but the results at the lowest $T^{*}$ and $\rho^{*}$ values begin to differ from the simulation results. This difference stems from the MPB theory beginning to break down in this region, as seen in the $g_{i}$ of Fig. 1(a). The negative slope at the higher $T^{*}$ is accurately predicted by the MPB theory.

The variation of $C_{i}^{*}$ with surface charge at $\rho^{*}=0.04$ for various $T^{*}$ is shown in Fig. 5. Due to the low-temperature limits on the theories, no MPB or DFT results are shown at

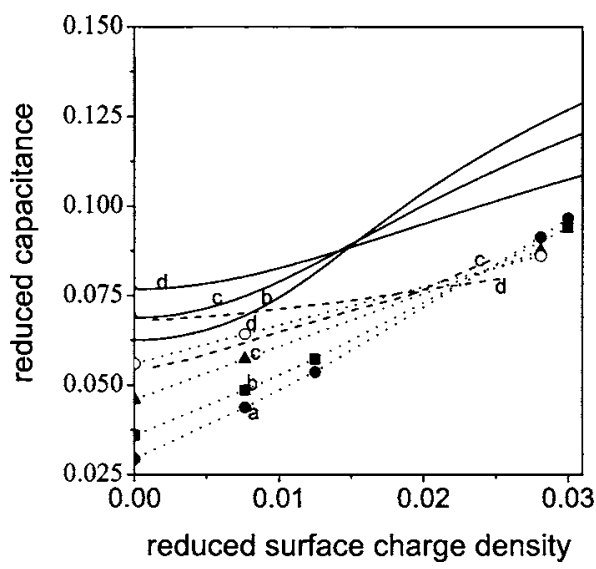

FIG. 5. Variation of the reduced capacitance $C_{i}^{*}$ with $\sigma^{*}$ at $\rho^{*}=0.04$ for (a) $T^{*}=0.06$, (b) $T^{*}=0.07$, (c) $T^{*}=0.08$, and (d) $T^{*}=0.1$ [solid line, MPB; dashed line, DFT; symbols, simulation (Ref. 5) points joined by dotted lines].

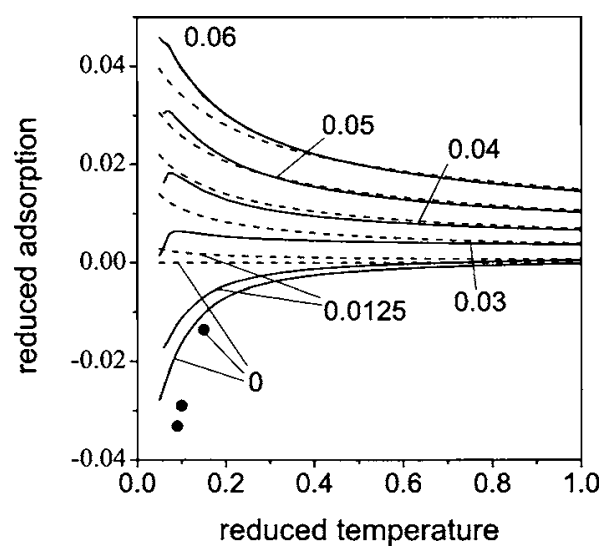

FIG. 6. The variation of adsorption $\Gamma$ with $T^{*}$ at $\rho^{*}=0.04$ for various $\sigma^{*}$ (solid line, MPB; dashed line, GC; solid circles, simulation (Ref. 5)).

the simulation value of $T^{*}=0.06$ and no DFT at $T^{*}=0.07$. The MPB results are poor compared with those of the DFT. The DFT has the correct linear behavior and is closer to the simulation results. However, all the theories predict that the lowering of the temperature produces a greater rate of change in $C_{i}^{*}$.

Some overall ion adsorption plots of

$$
\Gamma=\int \sum_{i} \rho_{i}^{*}\left[g_{i}(x)-1\right] d x
$$

as a function of $T^{*}$ are given in Fig. 6 at $\rho^{*}=0.04$ at various $\sigma^{*}$. As expected from the depleted distribution functions for $\sigma^{*} \sim 0$, the adsorption is negative for small surface charge indicating "drying" of the electrode. Increasing $\sigma^{*}$ leads to the counterions being attracted to the electrode and the adsorption becoming positive giving "wetting." At the higher values of $\sigma^{*}$ the MPB adsorption curves are similar to those of the GC theory. The behavior of the MPB adsorption curves at the lowest $T^{*}$ values for $0.02<\sigma^{*}<0.05$ is not in accord with those of the simulation results. ${ }^{5}$ It appears that this is due to the poor MPB representation of the singlet distribution function near the electrode as seen in Fig. 1(a). As noted earlier, negative adsorption is not restricted to near the critical region. However, as the critical point is approached, it is expected that the adsorption will be singular.

The negative adsorption at low surface charge can also be inferred from the contact theorem of Henderson et al., ${ }^{24}$

$$
k T \sum_{i} \rho_{i} g_{i}(d / 2)=p+\frac{2 \pi \sigma^{2}}{\varepsilon},
$$

where $p$ is the bulk pressure. Near the critical point $p$ is also small so that the sum of the contact values of the distribution functions is small implying negative adsorption. As $\sigma$ increases, the second term on the right-hand side of Eq. (12) becomes large, leading to positive adsorption.

\section{SUMMARY}

The MPB theory has been shown to be capable of predicting the correct capacitance behavior at low reduced temperatures and densities. At very low reduced temperatures the capacitance slope is positive which then changes to a 
negative slope as the temperature increases. This behavior is in agreement with the predictions of the MSA+mass action law, ${ }^{7}$ DFT, ${ }^{11}$ and simulation results. ${ }^{4-6}$ Negative adsorption is also predicted at these low reduced temperatures, although negative adsorption can occur at room temperatures for lowconcentration aqueous electrolytes at small surface charge. However, the negative adsorption is much greater at the low reduced temperatures and densities, with the adsorption probably diverging at the critical point. The MPB singlet distribution functions were found to contain a discontinuity in slope at $x=3 d / 2$ at very low $T^{*}$ and $\rho^{*}$. This unsatisfactory behavior in the $g_{i}$ is reflected in some of the poor predictions of the adsorption as $\Gamma$ depends on an integral over the $g_{i}$, whereas it minimally affects the capacitance. The discontinuity in the slope of distribution functions arises from the approximate solution of the fluctuation potential problem in the region close to the electrode. ${ }^{12}$ An improved solution of the fluctuation problem will correct the present shortcomings and provide an accurate MPB description of the RPM electrolyte in the vicinity of the critical point.

The influence of solvent effects on the capacitance behavior is the next important step. Simple models such as the solvent primitive model and the ion-dipole model of the electrolyte can be analyzed by a MPB approach. ${ }^{25,26}$ Ultimately a realistic solvent treatment using models such as TIPS4 or TIPS5, which presently the MPB approach cannot handle, is necessary. The recent works of Boda and co-workers, ${ }^{27-31}$ in which a simple treatment of the solvent is developed, are worth considering.

\section{ACKNOWLEDGMENTS}

One of the authors (I.B.B) acknowledges support of the NSF Grant No. 0137273. The authors are grateful to Dr. D. Boda for providing some simulation results.

\footnotetext{
${ }^{1}$ K. R. Painter, P. Ballone, M. P. Tosi, P. J. Grout, and N. H. March, Surf Sci. 133, 89 (1983).

${ }^{2}$ P. Ballone, G. Pastore, M. P. Tosi, K. R. Painter, P. J. Grout, and N. H. March, Phys. Chem. Liq. 13, 269 (1984).
}

${ }^{3}$ N. H. March and M. P. Tosi, Coulomb Liquids (Academic, London, 1984).

${ }^{4}$ D. Boda, D. Henderson, and K. Y. Chan, J. Chem. Phys. 110, 5346 (1999).

${ }^{5}$ D. Boda, D. Henderson, K. Y. Chan, and D. T. Wasan, Chem. Phys. Lett. 308, 473 (1999).

${ }^{6}$ D. Henderson, J. Mol. Liq. 92, 29 (2001).

${ }^{7}$ M. Holovko, V. Kapko, D. Henderson, and D. Boda, Chem. Phys. Lett. 341, 363 (2001).

${ }^{8}$ D. Henderson, D. Boda, and D. T. Wasan, Chem. Phys. Lett. 325, 655 (2000).

${ }^{9}$ L. Mier-Y-Teran, D. Boda, D. Henderson, and S. E. Quiñones-Cisneros, Mol. Phys. 99, 1323 (2001).

${ }^{10}$ D. Boda, D. Henderson, L. Mier-Y-Teran, and S. Sokolowski, J. Phys.: Condens. Matter 14, 11945 (2002).

${ }^{11}$ J. Resko-Zygmunt, S. Sokołowski, D. Henderson, and D. Boda, J. Chem. Phys. 122, 084504 (2005).

${ }^{12}$ C. W. Outhwaite and L. B. Bhuiyan, J. Chem. Soc., Faraday Trans. 2 79, 707 (1983).

${ }^{13}$ S. L. Carnie and G. M. Torrie, Adv. Chem. Phys. 46, 141 (1984).

${ }^{14}$ L. B. Bhuiyan and C. W. Outhwaite, Phys. Chem. Chem. Phys. 6, 3467 (2004).

${ }^{15}$ C. W. Outhwaite and L. B. Bhuiyan, J. Chem. Soc., Faraday Trans. 2 78, 775 (1982).

${ }^{16}$ S. Lamperski and C. W. Outhwaite, Langmuir 18, 3423 (2002).

${ }^{17}$ C. W. Outhwaite, L. B. Bhuiyan, and S. Levine, J. Chem. Soc., Faraday Trans. 2 76, 1388 (1980).

${ }^{18}$ C. W. Outhwaite, Condens. Matter Phys. 7, 719 (2004).

${ }^{19}$ L. B. Bhuiyan, C. W. Outhwaite, M. Molero, and E. González-Tovar, J. Chem. Phys. 100, 8301 (1994).

${ }^{20}$ E. González-Tovar and C. W. Outhwaite, Mol. Phys. 83, 1273 (1994).

${ }^{21}$ J. M. Romero-Enrique, G. Orkoulas, A. Z. Panagiotopoulos, and M. E. Fisher, Phys. Rev. Lett. 85, 4558 (2000).

${ }^{22}$ D. Henderson and D. Boda, J. Electroanal. Chem. (in press).

${ }^{23}$ D. Bratko, L. B. Bhuiyan, and C. W. Outhwaite, J. Phys. Chem. 90, 6248 (1986).

${ }^{24}$ D. Henderson, L. Blum, and J. L. Lebowitz, J. Electroanal. Chem. Interfacial Electrochem. 102, 315 (1979).

${ }^{25}$ S. Lamperski, C. W. Outhwaite, and L. B. Bhuiyan, Mol. Phys. 87, 1049 (1996).

${ }^{26}$ C. W. Outhwaite, Mol. Phys. 48, 599 (1983).

${ }^{27}$ D. Boda, J. Liszi, and I. Szalai, Mol. Phys. 85, 429 (1995).

${ }^{28}$ M. Valisko, D. Boda, J. Liszi, and I. Szalai, Phys. Chem. Chem. Phys. 3, 2995 (2001).

${ }^{29}$ M. Valisko, D. Boda, J. Liszi, and I. Szalai, Mol. Phys. 100, 3239 (2002).

${ }^{30}$ M. Valisko, D. Boda, J. Liszi, and I. Szalai, Mol. Phys. 101, 2309 (2003).

${ }^{31}$ M. Valisko and D. Boda, J. Phys. Chem. B 109, 6355 (2005). 